\title{
Graph Combinatorial Layout
}

National Cancer Institute

\section{Source}

National Cancer Institute. Graph Combinatorial Layout. NCI Thesaurus. Code C85854.

A type of graph similar to a radial layout, but compressed to fit rapidly-branching networks. 